\title{
Images of $B(k)$
}

by

Ronald C. Freiwald (St. Louis, Mo.)

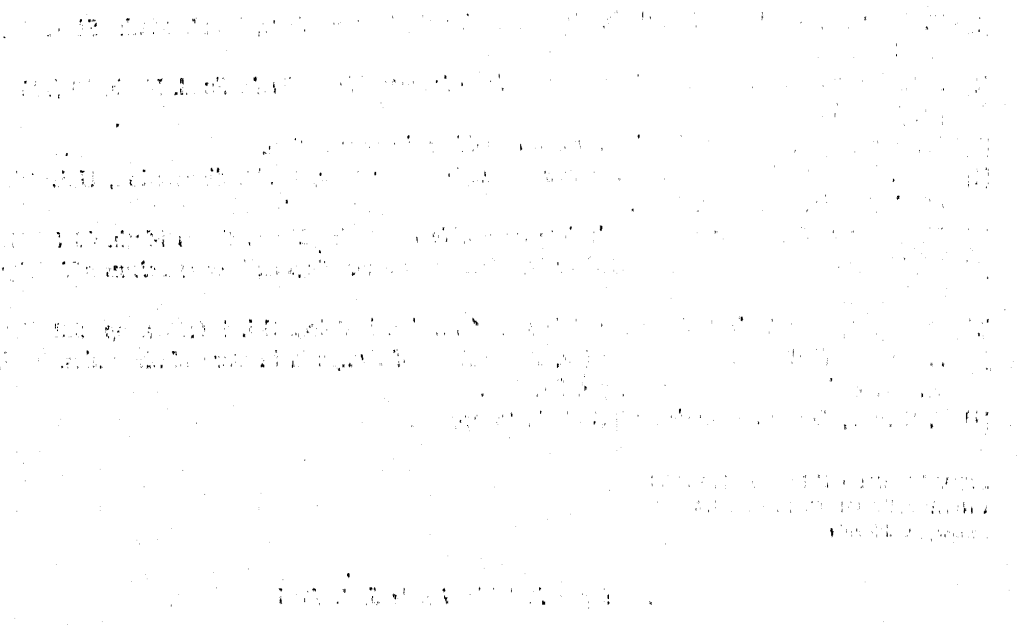

Abstract. There are a number of classical results concerning the existence of Borel isomorphisms of specified class between the space of irrational numbers and other separable (metric) absolute Borel sets. In this paper we consider analogous results for nonseparable (metric) absolute Borel sets. In particular, after proving a technical decomposition lemma for certain complete spaces, we characterize those complete spaces which are $(0,1)$ homeomorphs of $B(k)$, and prove that, roughly; every (metric) absolute Borel set of multiplicative class $\alpha+1$ which is of weight $k$ and not $\sigma \mathrm{LW}<k$ is, after the deletion of a "small" subset, a $(0, a+1)$ image of $B(k)$.

1. Preliminaries. All spaces considered are metrizable. The main facts about absolute Borel sets can be found in [5], [6], and [7]. We summarize some of the basic facts here for convenience.

Let $P$ denote one of the phrases "Borel", "Borel of additive class $.1<\alpha<\omega_{1}$ ", or "Borel of multiplicative class $1 \leqslant \alpha<\omega_{1}$ ". $X$ is said to be absolutely $P$ if $X$ is $P$ in every metric space in which it is embedded. This is equivalent to the statement that $X$ is $P$ in some complete space. $X$ is completely metrizable if and only if $X$ is absolutely of multiplicative class 1 , i.e., if and only if $X$ is an absolute $G_{\delta}$-set.

A bijection $f$ from $X$ to $Y$ is called a generalized homeomorphism of class $(\alpha, \beta)$ if whenever $V$ is open in $Y, f^{-1}(V)$ is of additive class $\alpha$ in $X$ and whenever $U$ is open in $X, f(U)$ is of additive class $\beta$ in $Y$. Such a map will be called an $(\alpha, \beta)$ map. for short. In particular, a $(0, \beta)$ map is continuous. $1 \ldots, \ldots+$

A space $X$ is said to be of $\sigma$-local weight $<k$ (abbreviated $\sigma \mathrm{LW}<k$ ), where $k$ is an infinite cardinal, if $X$ is the union of countably many subsets of local weight $<k$. Stone has shown $[6,7]$ that among absolute Borel sets, weight and $\sigma \mathrm{LW}<k$ are preserved by generalized homeomorphisms.

For $k$ an infinite cardinal, $B(\dot{k})$ denotes the product of countably many discrete spaces of cardinal $k$. In particular, $B\left(s_{0}\right)$ is the space of irrational numbers. In the classical theory of separable Borel sets [5] as well as in the nonseparable case $[6,7]$, the spaces $B(k)$ play a prominent role. In particular, Stone [7] has shown that an absolute Borel set.of weight $k$ is either $\sigma \mathrm{LW}<k$ or else contains a copy of $\dot{B}(\dot{k})$.

2. The main lemma. A space $X$ of weight $k$ is called weight-homogeneous if every nonempty open set has weight $k . \operatorname{In}[1]$, Comfort and Hager showed that if $X$ is 
a weight-homogeneous nonseparable metric space of weight $k$, then $X$ must contain a closed discrete set of cardinal $k$. This result is used in the proof of the following decomposition lemma.

LEMMa 1. Suppose $(X, d)$ is a weight-homogeneous metric space of weight $k>s_{0}$. Then there exists a sequence of families of well-ordered sets of cardinal $k$, denoted $A_{1}, A_{2}\left(a_{1}\right)$ (for each $\left.a_{1} \in A_{1}\right), \ldots, A_{n+1}\left(a_{1}, \ldots, a_{n}\right)$ (for each $n$-tuple $\left(a_{1}, \ldots, a_{n}\right)$ with $a_{1} \in A_{1}$ and $a_{i} \in A_{i}\left(a_{1}, \ldots, a_{i-1}\right)$ for $\left.i \geqslant 2\right), \ldots$, and, for each such $n$-tuple $\left(a_{1}, \ldots, a_{n}\right)$, there exist subsets $F\left(a_{1}, \ldots, a_{n}\right)$ and $D\left(a_{1}, \ldots, a_{n}\right)$ of $X$ such that:

1) $X=\bigcup\left\{D\left(a_{1}\right): a_{1} \in A_{1}\right\}$,

2) $D\left(a_{1}, \ldots, a_{n}\right)=F\left(a_{1}, \ldots, a_{n}\right)-\bigcup\left\{F\left(a_{1}, \ldots, a_{n-1}, a\right): \quad a \in A_{n}\left(a_{1}, \ldots, a_{n-1}\right)\right.$, $\left.a<a_{n}\right\}$ (where $A_{1}\left(a_{0}\right)=A_{1}$ ),

3) for $n>1, \quad D\left(a_{1}, \ldots, a_{n-1}\right)=\bigcup\left\{D\left(a_{1}, \ldots, a_{n-1}, a\right): a \in A_{n}\left(a_{1}, \ldots, a_{n-1}\right)\right\}$,

4) for each $n$, the sets $D\left(a_{1}, \ldots, a_{n}\right)$ are nonempty pairwise disjoint $F_{\sigma}$-sets and the family $\left\{D\left(a_{1}, \ldots, a_{n}\right): a_{n} \in A_{n}\left(a_{1}, \ldots, a_{n-1}\right), \ldots, a_{2} \in A_{2}\left(a_{1}\right), a_{1} \in A_{1}\right\}$ is $\sigma$-discrete,

5) for each $n, F\left(a_{1}, \ldots, a_{n}\right)$ is a closed set of diameter $\leqslant 1 / n$, and, for each $a_{n} \in A_{n}\left(a_{1}, \ldots, a_{n-1}\right), \bigcup\left\{F\left(a_{1}, \ldots, a_{n-1}, a\right): a \in A_{n}\left(a_{1}, \ldots, a_{n-1}\right), a<a_{n}\right\}$ is closed in $X$,

6) if $a_{1} \in A_{1}, a_{2} \in A_{2}\left(a_{1}\right), \ldots, a_{n} \in A_{n}\left(a_{1}, \ldots, a_{n-1}\right), \ldots$, then

$$
F\left(a_{1}\right) \supseteq D\left(a_{1}\right) \supseteq F\left(a_{1}, a_{2}\right) \supseteq D\left(a_{1}, a_{2}\right) \supseteq \ldots,
$$

7) each $D\left(a_{1}, \ldots, a_{n}\right)$ has weight $k$ and is weight-homogeneous.

Proof. Let $\mathfrak{B}=\{B(\alpha, n): \alpha<k, n=1,2 \ldots\}$ be a $\sigma$-discrete open base of cardinality $k$ for $X$, where, for each $n,\{B(\alpha, n): \alpha<k\}$ is discrete. We assume each $B(\alpha, n) \neq \varnothing$. Well-order $\mathfrak{B}$ by "last differences", i.e., $(\alpha, n)<(\beta, m)$ if either $n<m$ or else $n=m$ and $\alpha<\beta$. All sets of $n$-tuples occurring in the proof are to be ordered in this way.

Since $X$ is weight-homogeneous, $X$ contains a closed discrete set $P$ of cardinality $k$. For each $x \in X$, pick a set $B\left(\alpha_{x}, n_{x}\right) \in \mathfrak{B}$ which contains $x$, has diameter $<1$ and such that $\operatorname{cl} B\left(\alpha_{x}, n_{x}\right) \cap(P-\{x\})=\varnothing$. Let $F\left(\alpha_{x}, n_{x}\right)=\operatorname{cl} B\left(\alpha_{x}, n_{x}\right)$ and $D\left(\alpha_{x}, n_{x}\right)=F\left(\alpha_{x}, n_{x}\right)-U\left\{F\left(\alpha_{y}, n_{y}\right): y \in X,\left(\alpha_{y}, n_{y}\right)<\left(\alpha_{x}, n_{x}\right)\right\}$. Clearly,

$$
X=\bigcup\left\{D\left(\alpha_{x}, n_{x}\right): x \in X\right\} \text {. }
$$

The family $A_{1}=\left\{\left(\alpha_{x}, n_{x}\right): D\left(\alpha_{x}, n_{x}\right) \neq \varnothing\right\}$ has cardinality $k$ since, on the one hand, $|\mathfrak{B}|=k$, and, on the other, the family $\left\{D\left(\alpha_{x}, n_{x}\right): x \in X\right\}$ covers $X$ and each of its members meets $P$ in at most one point.

The sets $D\left(a_{1}\right)$ and $F\left(a_{1}\right)$ (for $\left.a_{1} \in A_{1}\right)$ have the required properties. Indeed, (1), (2), (3) and (6) are immediate. If $a_{1}, a_{1}^{\prime} \in A_{1}$, then $F\left(a_{1}\right)=\operatorname{cl} B\left(a_{1}\right)$ and $F\left(a_{1}^{\prime}\right)=\operatorname{cl} B\left(a_{1}^{\prime}\right)$ for some $B\left(a_{1}\right), B\left(a_{1}^{\prime}\right) \in \mathfrak{B}$. It is easy to check that if $a_{1} \neq a_{1}^{\prime}$, then $B\left(a_{1}\right) \neq B\left(a_{1}^{\prime}\right)$. From this, and the fact that $\bar{B}$ is $\sigma$-discrete, it follows that $\left\{F\left(a_{1}\right): a_{1} \in A_{1}\right\}$ and $\left\{D\left(a_{1}\right): a_{1} \in A_{1}\right\}$ are $\sigma$-discrete families, and then, from the definition of the ordering, that $\bigcup\left\{F(a): a<a_{1}\right\}$ is closed in $X$ for each $a_{1} \in A_{1}$.
The other items in (4) and (5) are now immediate. Finally, if $U$ is open in $X$ and $U \cap D\left(a_{1}\right) \neq \varnothing$, then

$$
\begin{aligned}
U \cap D\left(a_{1}\right) & =U \cap \operatorname{cl} B\left(a_{1}\right) \cap\left(X-\cup\left\{F(a): a<a_{1}\right\}\right) \\
& \supseteq U \cap B\left(a_{1}\right) \cap\left(X-\bigcup\left\{F(a) ; a<a_{1}\right\}\right) \neq \varnothing,
\end{aligned}
$$

since $X-\cup\left\{F(a): a<a_{1}\right\}$ is open. Therefore $U \cap D\left(a_{1}\right)$ has weight $k$ and $D\left(a_{1}\right)$ is weight-homogeneous.

Assume now that $n \geqslant 1$ and that we have defined for $i=1, \ldots, n$, the families of well ordered sets and the sets $F\left(a_{1}, \ldots, a_{i}\right)$ and $D\left(a_{1}, \ldots, a_{i}\right)$ as required. Since $D\left(a_{1}, \ldots, a_{n}\right)$ is weight-homogeneous of weight $k$, it contains a (relatively) closed discrete set $P\left(a_{1}, \ldots, a_{n}\right)$ of cardinality $k$. For each $x \in D\left(a_{1}, \ldots, a_{n}\right)$, pick an open set $U_{x}$ in $X$ which contains $x$ and such that

$$
U_{x} \cap D\left(a_{1}, \ldots, a_{n}\right) \cap\left(P\left(a_{1}, \ldots, a_{n}\right)-\{x\}\right)=\varnothing .
$$

For each $i=1, \ldots, n$, let $A_{i}=\bigcup\left\{F\left(a_{1}, \ldots, a_{i-1}, a\right): a<a_{i}, a \in A_{i}\left(a_{1}, \ldots, a_{i-1}\right)\right\}$ and let $F$ be the closed set $A_{1} \cup \ldots \cup A_{n}$. Using (5) and (6), we check that $D\left(a_{1}, \ldots, a_{n}\right)$ is disjoint from $F$. Pick an open set $O_{x}$ in $X$ which contains $x$ and misses $F$. Now choose a set $B\left(a_{1}, \ldots, a_{n},\left(\alpha_{x}, n_{x}\right)\right)$ from $\mathfrak{B}$ which contains $x$ and such that $\operatorname{cl} B\left(a_{1}, \ldots, a_{n},\left(\alpha_{x}, n_{x}\right)\right) \subseteq U_{x} \cap O_{x}$, and whose diameter is $<1 /(n+1)$. Let $F\left(a_{1}, \ldots, a_{n},\left(\alpha_{x}, n_{x}\right)\right)=\operatorname{cl}\left[B\left(a_{1}, \ldots, a_{n},\left(\alpha_{x}, n_{x}\right)\right) \cap D\left(a_{1}, \ldots, a_{n}\right)\right]$.

Since $D\left(a_{1}, \ldots, a_{n}\right) \subseteq F\left(a_{1}, \ldots, a_{n}\right)$, and because $F\left(a_{1}, \ldots, a_{n},\left(\alpha_{x}, n_{x}\right)\right) \subseteq O_{\dot{x}}$ (and therefore misses $F)$, it follows from (2) that $F\left(a_{1}, \ldots, a_{n},\left(\alpha_{x}, n_{x}\right) \subseteq D\left(a_{1}, \ldots, a_{n}\right)\right.$ Then .

so

$$
F\left(a_{1}, \ldots, a_{n},\left(\alpha_{x}, n_{x}\right)\right) \subseteq U_{x} \cap D\left(a_{1}, \ldots, a_{n}\right)
$$

$$
F\left(a_{1}, \ldots, a_{n},\left(\alpha_{x}, n_{x}\right)\right) \cap\left(P\left(a_{1}, \ldots, a_{n}\right)-\{x\}\right)=\varnothing
$$

Define

$$
\begin{aligned}
D\left(a_{1}, \ldots, a_{n},\left(\alpha_{x}, n_{x}\right)\right)=F\left(a_{1}, \ldots, a_{n},\left(\alpha_{x}, n_{x}\right)\right)- \\
-U\left\{F\left(a_{1}, \ldots, a_{n},\left(\alpha_{y}, n_{y}\right)\right):\left(\alpha_{y}, n_{y}\right)<\left(\alpha_{x}, n_{x}\right), y \in D\left(a_{1}, \ldots, a_{n}\right)\right\} .
\end{aligned}
$$

Clearly $D\left(a_{1}, \ldots, a_{n}\right)=\bigcup\left\{D\left(a_{1}, \ldots, a_{n},\left(\alpha_{x}, n_{x}\right)\right): x \in D\left(a_{1}, \ldots, a_{n}\right)\right\}$. Let

$$
A_{n+1}\left(a_{1}, \ldots, a_{n}\right)=\left\{\left(\alpha_{x}, n_{x}\right): x \in D\left(a_{1}, \ldots, a_{n}\right), D\left(a_{1}, \ldots, a_{n},\left(\alpha_{x}, n_{x}\right)\right) \neq \varnothing\right\} .
$$

As in the case $n=1,\left|A_{n+1}\left(a_{1}, \ldots, a_{n}\right)\right|=k$.

It is easy to verify that (1), (2), (3) and (6) hold for these sets. As before, we claim that if $\left(a_{1}, \ldots, a_{n+1}\right)$ and $\left(a_{1}^{\prime}, \ldots, a_{n+1}^{\prime}\right)$ from $A_{n+1}\left(a_{1}, \ldots, a_{n}\right)$ are distinct, then $B\left(a_{1}, \ldots, a_{n+1}\right) \neq B\left(a_{1}^{\prime}, \ldots, a_{n+1}^{\prime}\right)$. For suppose $a_{j}<a_{j}^{\prime}$ and $a_{i}=a_{i}^{\prime}$ for each $i<j$. Then, since $\operatorname{cl} B\left(a_{1}^{\prime}, \ldots, a_{n+1}^{\prime}\right) \subseteq O_{x}$ for some $x \in D\left(a_{1}^{\prime}, \ldots, a_{n}^{\prime}\right)$, we get that

$$
\operatorname{cl} B\left(a_{1}^{\prime}, \ldots, a_{n+1}^{\prime}\right) \cap \bigcup\left\{F\left(a_{1}^{\prime}, \ldots, a_{j-1}^{\prime}, a\right): a<a_{j}^{\prime}\right\}=\varnothing ;
$$


so that, by (6),

$\varnothing=\operatorname{cl} B\left(a_{1}^{\prime}, \ldots, a_{n+1}^{\prime}\right) \cap F\left(a_{1}^{\prime}, \ldots, a_{j-1}^{\prime}, a_{j}\right) \supseteq \operatorname{cl} B\left(a_{1}^{\prime}, \ldots, a_{n+1}^{\prime}\right) \cap F\left(a_{1}, \ldots, a_{n+1}\right)$. But $F\left(a_{1}, \ldots, a_{n+1}\right) \subseteq \operatorname{cl} B\left(a_{1}, \ldots, a_{n+1}\right)$, so if $B\left(a_{1}, \ldots, a_{n+1}\right)=B\left(a_{1}^{\prime}, \ldots, a_{n+1}^{\prime}\right)$, then $\varnothing=F\left(a_{1}, \ldots, a_{n+1}\right)=D\left(a_{1}, \ldots, a_{n+1}\right)$, a contradiction. From this and the fact that $\overline{\mathfrak{B}}$ is $\sigma$-discrete, it follows that $\left\{D\left(a_{1}, \ldots, a_{n+1}\right): a_{n+1} \in A_{n}\left(a_{1}, \ldots, a_{n}\right), \ldots, a_{1} \in A_{1}\right\}$ and $\left\{F\left(a_{1}, \ldots, a_{n}, a\right): a<a_{n+1} \in A_{n+1}\left(a_{1}, \ldots, a_{n}\right)\right\}$ are $\sigma$-discrete, and, from the definition of the ordering, that the union of the latter family is closec. The other items in (4) and (5) now follow immediately.

- Finally, to verify (7), suppose $U$ is open in $X$ and $U \cap D\left(a_{1}, \ldots, a_{n+1}\right) \neq \varnothing$. Letting $O$ denote the open set $X-\cup\left\{F\left(a_{1}, \ldots, a_{n}, a\right): a<a_{n+1}\right\}$, we get $\varnothing \neq U \cap D\left(a_{1}, \ldots, a_{n+1}\right)=U \cap \operatorname{cl}\left[B\left(a_{1}, \ldots, a_{n+1}\right) \cap D\left(a_{1}, \ldots, a_{n}\right)\right] \cap O$, so $U \cap O \cap B\left(a_{1}, \ldots, a_{n+1}\right) \cap D\left(a_{1}, \ldots, a_{n}\right) \neq \varnothing$; since this is a nonempty open set in $D\left(a_{1}, \ldots, a_{n}\right)$ it has weight $k$ by $(7)$ of the induction hypothesis; hence $D\left(a_{1}, \ldots, a_{n+1}\right)$ is weight-homogeneous of weight $k$

3. Images of $B(k)$. The following theorem characterizes the complete metric spaces which are $(0,1)$ images of $B(k)$.

THEOREM 2. Suppose $X$ is a complete metric space of infinite weight $k$. Then there exists a $(0,1)$-map from $B(k)$ onto $X$ if and only if $X$ is weight-homogeneous.

Proof. If $k=\kappa_{0}$, then every nonempty open set in $X$ must be dense-in-itself and therefore uncountable. It then follows from a classical theoren [5] that $X$ is a $(0,1)$ homeomorph of $B\left(\aleph_{0}\right)$.

If $k>s_{0}$, decompose $X$ according to Lemma 1 , making the index sets disjoint. Let $Y=\left\{\left(a_{1}, \ldots, a_{n}, \ldots\right): a_{1} \in A_{1}, \ldots, a_{n} \in A_{n}\left(a_{1}, \ldots, a_{n-1}\right), \ldots\right\}$. Since each index set has cardinality $k, Y$, with the "first difference" metric, is homeomorphic to $B(k)$.

For each $y=\left(a_{1}, \ldots, a_{n}, \ldots\right) \in Y, \bigcap_{n=1}^{\infty} D\left(a_{1}, \ldots, a_{n}\right) \supseteq \bigcap_{n=2}^{\infty} F\left(a_{1}, \ldots, a_{n}\right)$ contains a single point $f(y)$. It follows from (1), (3), and (4) of Lemma 1 that $f$ is a bijection from $Y$ to $X$.

If $y=\left(a_{1}, \ldots, a_{n}, \ldots\right)$ and $y^{\prime}=\left(a_{1}^{\prime}, \ldots, a_{n}^{\prime}, \ldots\right)$ are at distance $\leqslant 1 / n<1$ in $Y$, then $f(y)$ and $f\left(y^{\prime}\right)$ are both in $D\left(a_{1}, \ldots, a_{n-1}\right)$ which has diameter $<1 /(n-1)$. Hence $f$ is (uniformly) continuous.

Let

$$
\begin{aligned}
S_{1}=A_{1}, S_{2}= & \left\{\left(a_{1}, a_{2}\right): a_{2} \in A_{2}\left(a_{1}\right)\right\}, \ldots \\
& \ldots, S_{n}=\left\{\left(a_{1}, \ldots, a_{n}\right): a_{n}^{\prime} \in A_{n}\left(a_{1}, \ldots, a_{n-1}\right), \ldots, a_{1} \in A_{1}\right\}
\end{aligned}
$$

and let $V\left(a_{1}, \ldots, a_{n}\right)$ be the (basic) open set in $Y$ consisting of all points with initial coordinates $\left(a_{1}, \ldots, a_{n}\right)$. Then, if $O$ is open in $Y$,

$$
O=\bigcup\left\{V\left(a_{1}\right): a_{1} \in S_{1}^{\prime}\right\} \cup \ldots \cup \cup\left\{V\left(a_{1}, \ldots, a_{n}\right):\left(a_{1}, \ldots, a_{n}\right) \in S_{n}^{\prime}\right\} \cup \ldots,
$$

where $S_{i}^{\prime} \subseteq S_{i}$. Clearly, $f\left(V\left(a_{1}, \ldots, a_{n}\right)\right)=D\left(a_{1}, \ldots, a_{n}\right)$, so

$$
f(0)=\bigcup\left\{D\left(a_{1}\right): a_{1} \in S_{1}^{\prime}\right\} \cup \ldots \cup \cup\left\{D\left(a_{1}, \ldots, a_{n}\right):\left(a_{1}, \ldots, a_{n}\right) \in S_{n}^{\prime}\right\} \cup \ldots
$$

But since each family of sets $\left\{D\left(a_{1}, \ldots, a_{n}\right):\left(a_{1}, \ldots, a_{n}\right) \in S_{n}^{\prime}\right\}$ is a $\sigma$-discrete family of $F_{\sigma}$-sets, it follows that $f(0)$ is an $F_{\sigma}$-set in $X$; so $f$ is a $(0,1)$-map.

On the other hand, if $X$ is a complete metric space (or even any absolute Borel set) of weight $k$, and if $f: B(k) \rightarrow X$ is a $(0, \alpha)$-map, then $X$ must be weight homogeneous. Indeed, if 0 is a nonempty open set in $X$, then the open set $f^{-1}(0)$ must have weight $k$. But the absolute Borel sets $O$ and $f^{-1}(0)$ are generalized homeomorphic and therefore have the same weight.

We remark that Stone [6] showed that if $X$ is a complete metric space of weight $k$ then there is a $(0,1)$-map from a closed subset $A$ of $B(k)$ onto $X$. The additiona assumption of weight-homogeneity allows us to take $A=B(k)$.

Corollary 3. Suppose $X$ is a complete metric space of infinite weight $k$. Then there exists $a(0,1)$-map from $B(k)$ onto $X$ if and only if no nonempty open set in $X$ is $\sigma \mathrm{LW}<k$.

Proof. If no nonempty open set in $X$ is $\sigma \mathrm{LW}<k$, then $X$ is weight-homogeneous. On the other hand, if $X$ is weight-homogeneous and $O$ is a nonempty open set, then $O$, by Theorem 2, is generalized homeomorphic to $B(k)$ and therefore is not $\sigma \mathrm{LW}<k$.

Hausdorff showed [3] that every separable absolute Borel set of multiplicative class $\alpha+1 \geqslant 2$ in which every point is a condensation point is a $(0, \alpha)$ homeomorph of $B\left(s_{0}\right)$. Since a separable set is $\sigma \mathrm{LW}<s_{0}$ if and only if it is countable, Corollary 3 generalizes Hausdorff's result, for complete spaces, to the nonseparable case. A further step toward a full generalization of Hausdorff's result is in Theorem 6. Corollary 3 also generalizes the classical result that every uncountable complete separable metric space is, after the deletion of a countable set, a $(0,1)$ homeomorph of $B\left(s_{0}\right)$.

The following corollaries all generalize classical results. which can be found in [5].

COROLlary 4. Let $X$ be a metric space of infinite weight $k$. Then there is $a(0,1)$ map $f$ from a subset $A$ of $B(k)$ onto $X$.

Proof. Let $S$ be the metric space consisting of $k$ copies of $[0,1]$ identified at $O$, with the "shortest path" metric, and let $P$ be the product of countably many copies of $S$. Then $P$ is a completely metrizable, weight-homogeneous space of weight $k$, and therefore there is a $(0,1)$-map $g: B(k) \rightarrow P$. Since $P$ is universal for metric spaces of weight $k$ [4], we may assume $X \subseteq P$, and put $A=g^{-1}(X)$ and $f=g \mid A$.

COROLLARY 5. If $X$ and $Y$ are complete, weight-homogeneous metric spaces of weight $k$, then there is a $(1,1)$-map $h: X \rightarrow Y$.

Proof. Let $h=f \circ g^{-1}$, where $g$ and $f$ are $(0,1)$-maps from $B(k)$ to $X$ and $Y$ respectively.

The following theorem moves in the direction of a full generalization of Hausdorff's result. It also generalizes to the nonseparable case and to higher Borel classes the classical result that if $X$ is an uncountable complete separable metric space, then there exists a countable set $C \subseteq X$ and a $(0,1)$-map of $B\left(\kappa_{0}\right)$ onto $X-C$. 
TheoREM 6. Let $X$ be an absolute Borel set, of multiplicative class $\alpha+1 \geqslant 2$ which has weight $k$, and is not $\sigma \mathrm{LW}<k$. Then there is a set $C \subseteq X$ of additive class $\alpha$ and $\sigma \mathrm{LW}<k$ and $a(0, \alpha+1)$-map of $B(k)$ onto $X-C$.

Proof. By a theorem of Hansell [2], there exists a $(0, \alpha)$-map $g: A \rightarrow X$ where $A$ is a closed subset of $B(k)$. Since $X$ is not $\sigma \mathrm{LW}<k$, neither is $A$. Let $B$ be the largest open subset of $A$ which is $\sigma \mathbf{L W}<k$ ( $B$, for example, can be described as the set of all points of $A$ which are not in a homeomorph of $B(k)$ in $A$ [7]). Then $A-B$ is a completely metrizable space of weight $k$ in which no nonempty (relatively) open set is $\sigma \mathrm{LW}<k$. Hence, by Corollary 3 , there is a $(0,1)$-map $h: B(k) \rightarrow A-B$. Then $f=(g \mid(A-B) \circ h)$ is a $(0, \alpha+1)$-map of $B(k)$ onto $X-C$, where $C=g(B)$. Since $B$ is open in $A$ and is $\sigma \mathrm{LW}<k, C$ is of additive class $\alpha$ in $X$ and has $\sigma \mathrm{LW}<k$.

\section{References}

[1] W. Comfort and A. Hager, Metric spaces without large closed discrete sets, to appear.

12] R. Hansell, Borel measurable mappings for non-separable metric spaces, Thesis, University of Rochester, 1969.

[3] F. Hausdorff, Die schlichten stetigen Bilder des Nullraums, Fund. Math. 29 (1937), pp. 151-158.

[4] H. Kowalsky, Einbettung metrischer Räume, Arch. Math. 8 (1957), pp. 336-339.

[5] K Kuratowski, Topology, Yol, I, New York-London-Warszawa 1966.

[6] K. Stone, Non-separable Borel sets, Dissertationes Math. 28 (1962).

[6] A. Stone, Non-separable Borel sets, Dissertationes Math. 28 (1962).

WASHINGTON UNIVERSITY

St. Louis, Missouri

Accepté par la Rédaction le 21. 1. 1976

\section{LIVRES PUBLIES PAR LINSTITUT MATHEMATIQUE DE L'ACADEMIE POLONAISE DES SCIENCES}

S. Banach, Oeuvres, Vol. I, 1967, p. 381.

S. Mazurkiewicz, Travaux de topologie et ses applications, 1969, p. 380.

W. Sierpiński, Oeuvres choisies, Vol. I, 1974, p. 300; Vol. II, 1975, p. 780; Vol. III, 1976, p. 688. J. P. Schauder, Oeuvres, 1978, p. 487.

Proceedings of the Symposium to honour Jerzy Neyman, 1977, p. 349.

\section{MONOGRAFIE MATEMATYCZNE}

41. H. Rasiowa and R. Sikorski, The mathematics of metamathematics, 3-ème éd, corrigée 1970 , p. 520.

43. J. Szarski, Differential inequalities, 2-ème éd., 1967, p. 256.

44. K. Borsuk, Theory of retracts, 1967, p. 251.

45. K. Maurin, Methods of Hilbert spaces, 2-ème éd., 1972, p. 552.

47. D. Przeworska-Rolewicz and S. Rolewicz, Equations in linear spaces, 1968, p. 380

50. K. Borsuk, Multidimensional analytic geometry, 1969, p. 443.

51. R. Sikorski, Advanced calculus. Functions of several variables, 1969, p. 460.

52. W. Ślebodziński, Exterior forms and their applications, 1970, p. 427.

53. M. Krzyżański, Partial differential equations of second order, I, 1971, p. 562.

54. M. Krzyżański, Partial differential equations of second orđer, II, 1971, p. 407.

57. W. Narkiewicz, Elementary and analytic theory of algebraic numbers, 1974, p. 630

58. C. Bessaga and A. Pełczyński, Selected topics in infinite-dimensional topology, 1975, p. 353.

59. K. Borsuk, Theory of shape, 1975 , p. 379.

60. R. Engelking, General topology, 1977, p. 626

\section{DISSERTATIONES MATHEMATICAE}

CLII. K. Szymiczek, Quadratic forms over fields, 1977, p. 67,

CLIII. W. Mlak, Dilations of Hilbert space operators (general theory), 1977, p. 65.

CLIV. V. M. Bogdan and R. A. Oberle, Topological rings of sets and the theory of vector measures, 1978. p. 74 .

\section{BANACH CENTER PUBLICATIONS}

Vol. 1. Mathematical control theory, 1976, p. 166.

Vol. 2. Mathematical fundations of computers science, 1977, p. 260

Vol. 3. Mathematical models and numerical methods, 1978, p. 391.

Vol. 4 Approximation theory (en préparation). 